\title{
Wohlfahrtsökonomische und systemtheoretische Ansätze zur Bestimmung von Gemeinwohl
}

\section{Zur inhaltlichen Unbestimmtheit von „Gemeinwohl“}

Die griechische Staatslehre gilt allgemein als eine wichtige Wurzel wenigstens der europäischen Gemeinwohlkonzeptionen. Bei Plato weiß der Philosophen-König, was gerecht ist und der Allgemeinheit dient. Die von Plato geforderte Philosophenherrschaft unterstellt, daß das Wohl des Gemeinwesens von der Fähigkeit des Herrschers zur Erkenntnis des Guten und von seiner Motivation, seiner Liebe zur Gerechtigkeit abhängt. Auch bei Aristoteles weiß der gute Monarch, was der Allgemeinheit dient, und sucht es $\mathrm{zu}$ verwirklichen, und auch hier kommt es auf die Handlungsorientierung der Herrscher an: So unterscheidet sich die (gute) Staatsform Monarchie von der Tyrannis dadurch, daß der Herrscher im einen Fall den Nutzen der Untertanen, im anderen den eigenen Nutzen verfolgt. ${ }^{1}$ Plato wie Aristoteles unterscheiden also zwischen Eigennutz und Gemeinnutz, aber der gemeine Nutzen wird letztlich am individuellen Wohl der Untertanen, der Bürger festgemacht. Auch Kaufmann betont, die auf Aristoteles aufbauende politische Theorie habe keinen Widerspruch zwischen Partialinteressen und Gemeininteresse gesehen, weil das Wohl des Ganzen, der Polis, mit dem Wohl ihrer Mitglieder gleichgesetzt wurde. ${ }^{2}$ Im Europa der Renaissance griff man auf die klassischen Vorstellungen zurück. Zu den Fürstenpflichten gehört die Sicherung von Frieden und Recht; damit soll der "gute" Herrscher das Wohl seiner Untertanen verwirklichen. Noch später wird Gemeinwohl zum ,staatsbegründenden Ziel“ und zur Norm, an die der Herrscher und sein Herrschaftsanspruch gebunden sind. ${ }^{3}$ Worin das gemeine Wohl besteht und ob es in Konflikt mit den individuellen Interessen der Gesellschaftsmitglieder geraten kann, wurde dabei offenbar wenig problematisiert. Auch wenn die „gute Ordnung“ in der Wirklichkeit nicht erreicht wurde, erschien die gleichzeitige Maximierung des individuellen und des gemeinen Wohls möglich.

\footnotetext{
Vgl, Nunner-Winkler 1994.

Vgl. Kaufmann 1994, S. 358.

Siehe dazu Münkler/Fischer 1999.
} 
Die liberale Kritik des absolutistischen Staats veranlaßte zunächst, zwischen individueller Wohlfahrt in Form der bürgerlichen Freiheiten und der Notwendigkeit der Gewährleistung innerer Ordnung und äußerer Unabhängigkeit zu unterscheiden, „ohne beiden das gleiche Ethos zuzumuten". ${ }^{4}$ Diese Differenzierung führte jedoch in der Folgezeit nicht dazu, daß nun mögliche Konflikte zwischen beiden „Ethiken“ in den Mittelpunkt des Interesses rückten. Die Konzentration auf die beiden Staatsfunktionen der inneren Ordnung und äußeren Sicherheit bewirkte nach Kaufmann vielmehr, daß die Frage nach der individuellen Wohlfahrt aus dem politischen Diskurs ausgeschlossen wurde, solange nur jedem Individuum das Recht auf Verfolgung des eigenen Glücks erhalten blieb. In der Folge einer solchen individualistischen Argumentation wurde der Wohlfahrtsbegriff auf das private Glück reduziert; so wurde Wohlfahrt schließlich mit der Summe individueller Bedürfnisbefriedigung gleichgesetzt. Und kritisch fragt Kaufmann: „Woher aber nahm die liberale politische Theorie die Überzeugung, daß dieses individualistische Programm der Glückssuche nicht nur die individuellen, sondern auch den gemeinen Nutzen fördere? ${ }^{45}$ Davon hatte sie wohl Adam Smith überzeugt: Bei ihm entstand das Gemeinwohl, nun als ökonomischer Wohlstand aufgefaßt, automatisch als Nebenfolge des egoistischen Handelns der einzelnen. So wurde der Wohlfahrtsdiskurs ökonomisiert und das Gemeinwohlkonzept individualisiert.

Relativ unbestimmt blieb der Inhalt des auf ein größeres Ganzes bezogenen Gemeinwohls auch in dem im Mittelalter auf der Ebene selbständiger Kommunen bzw. Städte verbreiteten Konzept des ,gemeinen Nutzes“. ${ }^{\text {" }}$ Nur indirekt läßt sich aus den Entscheidungen, die mit dem ,gemeinen“ oder auch „Stadt"-Nutzen argumentieren, der Inhalt der sie leitenden Gemeinwohlvorstellungen ableiten: Es geht um Ordnungsstiftung, um die Mehrung des Wohlstands und um die Autonomie des Gemeinwesens. Versuche, das Gemeinwohl inhaltlich näher zu bestimmen, scheitern tatsächlich in der Regel an den von Claus Offe ${ }^{7}$ herausgearbeiteten Schwierigkeiten, die sozialen, zeitlichen und sachlichen Bezugspunkte einer Gemeinwohldefinition zu fixieren. Zwar lieBen sich die Schwierigkeiten bei der Bestimmung des sozialen Bezugspunkts noch relativ einfach lösen, indem man sich - wie das hier geschehen soll - auf das jeweils umfassendste, ein integriertes Sozialgebilde darstellende System beschränkt. Dieses System ist heute in den meisten Fällen eine national verfaßte Gesellschaft, kann aber z.B. auch der Clan sein, wenn ein Staat als reales Identifikationsobjekt und durchsetzungsfähige Instanz fehlt. Damit bleiben aber immer noch die Probleme, die mit den beiden anderen für eine inhaltliche Gemeinwohlbestimmung nötigen Dimensionen verbunden sind: Fristigkeit (geht es um das Wohl des Gemeinwesens heute oder in einer zu bestimmenden Zukunft?) und sachlicher Gehalt (worin besteht das Wohl des Gemeinwesens?).

Angesichts dieser Schwierigkeiten gehen die meisten zur Zeit gängigen sozialwissenschaftlichen Ansätze davon aus, daß es für die Bestimmung von Gemeinwohl auf der Ebene ganzer Gesellschaften keinen objektiven Maßstab gibt. Vielmehr scheint generell

\footnotetext{
Kaufmann 1994, S. 358.

Ebd.

Vgl. Blickle 2001.

$\mathrm{Vgl}$. Offe, in diesem Band.
} 
akzeptiert, daß jeweils in einem politischen Prozeß Einverständnis über das hergestellt werden muß, was von den Mitgliedern der Gesellschaft als erstrebenswerter Zustand für diese ihre Gesellschaft angesehen wird - gleichgültig, ob man sich dabei nun auf ein eher utopisches Diskursmodell stützt, bei dem durch rationale Argumentation am Ende ein inhaltlicher Konsens gefunden wird, oder ob der Zielzustand als Kompromiß verschiedener Auffassungen bestimmt wird. In beiden Fällen ist ein bestimmtes Verfahren die Basis der Gemeinwohlbestimmung: es sind prozedurale, keine inhaltlichen Ansät$\mathrm{ze}^{8}$ Auch ich habe in anderem Zusammenhang schon betont, daß es keinen Konsens darüber gebe, worin das bonum commune besteht.

„Das immer nur in Teilaspekten konkret formulierte Gemeinwohl ist - immer vorläufiges und revidierbares - Ergebnis einer fortdauernden Auseinandersetzung, an der neben staatlichen Instanzen andere Verbände und sonstige nicht-staatliche Akteure, Sachverständige in Beiräten und vor allem die durch die Medien repräsentierte Öffentlichkeit beteiligt sind. “9

Auch wenn man nicht explizit unterstellt, daß es im rationalen Diskurs der Gesellschaftsmitglieder um die Identifikation und Erfüllung von Systeminteressen geht, verleitet eine prozedurale Betrachtungsweise leicht zu der Annahme, daß die Einbeziehung von möglichst allen Betroffenen und Interessenten in einen politischen Entscheidungsprozeß nicht lediglich zum Interessenausgleich, sondern zu einer dem Gemeinwohl dienenden Lösung führt. Im Extremfall wird damit mehr Bürgerbeteiligung umstandslos mit mehr Gemeinwohl gleichgesetzt. Dabei setzt man implizit oder explizit auf das Funktionieren eines höchst voraussetzungsvollen Mechanismus des Orientierungswandels, der in der Interaktion von Vertretern anfänglich partikularistischer Interessen dazu führt, daß sie gemeinsam das Wohl des größeren Ganzen verfolgen. Derartige Prozesse des Orientierungswandels gibt es, und sie sind von Sozialwissenschaftlern verschiedentlich empirisch nachgewiesen worden. ${ }^{10}$ Die Achillesferse prozeduraler Ansätze zur Gemeinwohlbestimmung bleibt jedoch ihre Maßstablosigkeit: sie erlauben nicht, das Wohl des Gemeinwesens, die Systemrationalität des in einem solchen Prozeß schließlich gefundenen Konsenses zu beurteilen.

Die beiden hier zu behandelnden Ansätze dagegen, die jeder für sich auf eine ehrwürdige Denktradition verweisen können, bieten objektive Maßstäbe zur Bestimmung des Gemeinwohls an. Dabei wenden sich beide gegen eine stillschweigende Gleichsetzung bei der Beurteilung des ,Wohls“ von Individuen und sozialen Systemen und versuchen, mehr oder weniger präzise zu fassen, worin das Gemeinwohl besteht. Die beiden Ansätze unterscheiden sich jedoch in einem ganz wesentlichen Punkt: Wohlfahrtsökonomische Ansätze finden ihren Maßstab in der individuellen Bedürfniserfüllung, während die funktionalistische Systemtheorie ihren Maßstab in der Erfüllung grundlegender Systembedürfnisse findet. Beide Positionen sind dabei gleichermaßen eindimensional: Ob der relevante Nutzen auf der Systemebene oder der Individualebene bestimmt wird - in beiden Fällen wird die Möglichkeit, ja Wahrscheinlichkeit einer Spannung bzw. eines Konflikts zwischen den beiden Maßstäben nicht thematisiert.

\footnotetext{
Vgl. Schuppert 2002; von Alemann/Heinze/Wehrhöfer 1999.

Mayntz 1992, S. 18, 32.

${ }^{10}$ Vgl. Mayntz 1997, S. 249-259.
} 


\section{Wohlfahrtsökonomische Ansätze}

Die klassische, auch paretianisch genannte Wohlfahrtsökonomie summiert den individuellen Nutzen der Systemmitglieder und setzt dies mit dem Gemeinwohl gleich. Vorausgesetzt wird dabei entweder, daß der erstrebte Nutzen für alle Individuen gleich ist, z.B. Einkommen, oder sich doch auf einer einheitlichen Nutzendimension abbilden läßt. Im ersten Fall schien sich das Sozialprodukt als quantitatives Maß des Gesamtnutzens anzubieten. Diese Operationalisierung ist aber bald und grundlegend kritisiert worden. Nach Albert (1998) ist die Konkretisierung von Gemeinwohl als Maximierung des Sozialprodukts vor allem wegen der Vernachlässigung vieler individueller Werte bei der Messung des Sozialprodukts und der Ungleichwertigkeit der finanziell gleich bewerteten Transaktionen unhaltbar. Ob Spielkasino, Krankenhaus oder Bordell: Umsatz ist gleich Umsatz. Aber auch unabhängig von der problematischen Operationalisierung als Sozialprodukt bot die utilitaristische Wohlfahrtsdefinition als Summe individueller Nutzen Anlaß zur Kritik. Diese Kritik bezieht sich vor allem (1) auf die Vernachlässigung der Unterschiedlichkeit individueller Präferenzen und auf die Vernachlässigung (2) der zeitlichen und (3) der sozialen Dimension in der Verteilung des Gesamtnutzens.

Um die Schwächen der paretianischen Wohlfahrtsökonomik zu überwinden, wurde die Konzeption der sozialen Wohlfahrtsfunktion entwickelt. Die Theorie von social choice geht von der Existenz unterschiedlicher Präferenzordnungen aus und sucht nach einem Algorithmus, der es erlaubt, hieraus einen kollektiven Nutzen abzuleiten, d.h. sie will „das Problem einer adäquaten sozialen Ordnung mit Hilfe eines entscheidungslogischen Kalküls [...] lösen, der an die Präferenzstrukturen der Individuen anknüpft und es erlaubt, aus ihnen eine kollektive Präferenz abzuleiten."12 Bezugspunkt bleibt aber auch hier der individuelle Nutzen: es geht um eine Entscheidung, die das mögliche Höchstmaß an Befriedigung der ungleichen individuellen Präferenzen bringt. Auf diese Weise wird das Gemeinwohl zu einer mathematischen Fiktion.

Tatsächlich hat Kenneth Arrow gezeigt, daß es nicht möglich ist, die verschiedenen individuellen Präferenzen der Bürger eines Landes in eine logisch widerspruchsfreie kollektive Präferenzordnung zu überführen (Theorem der Unmöglichkeit der Existenz einer sozialen Wohlfahrtsfunktion). Benutzt man überdies als Aggregationsverfahren eine einfache Mehrheitsregel, dann müssen höchst unrealistische, restriktive Bedingungen erfüllt sein, um zu vermeiden, daß die Aggregation zu widersprüchlichen kollektiven Präferenzen führt. ${ }^{13}$ Fritz Scharpf stellt denn auch fest, es sei empirisch erwiesen, daß eine Aggregation unterschiedlicher individueller Präferenzen durch Mehrheitsentscheidung chaotisch-instabile Lösungen produziert und auch aus der Sicht der Beteiligten suboptimal ist - und das bedeute ja wohl, daß das utilitaristische Gemeinwohlkonzept des Netto-Gesamtnutzens noch keine gangbare Annäherung an das Gemeinwohl brächte. $^{14}$

\footnotetext{
1 Vgl. Scharpf 1997, S. 90.

12 Albert 1998, S. 13.

13 Vgl. Gaertner 1998.

14 Vgl. Scharpf 1994, S. 385 f.
} 
Ein weiterer, schon kurz erwähnter Schwachpunkt des wohlfahrtsökonomischen Ansatzes ist, daß er nur die Größe der Nutzensumme betrachtet und die Verteilung dieser Summe auf die Systemmitglieder völlig vernachlässigt. „Modern welfare economics declares itself incompetent to judge the distributive issues. [...] One reason for this is related to the conceptual difficulty of collapsing values differing in type and in their temporal, local, and personal incidence into a single measure of aggregate utility. " 15 Amartya Sen hat deshalb (nach Gaertner) ein Armutsmaß eingeführt, das die Zahl der Armen und das Ausmaß der Armut berücksichtigt; dieses Armutsmaß soll in die $\mathrm{Be}$ stimmung des kollektiven Nutzens eingehen. ${ }^{16}$ Das liegt ganz auf der Linie von Rawls, der im zweiten seiner beiden Gerechtigkeitsprinzipien konstatiert, daß eine ungleiche Verteilung von Gütern (Einkommen, Nutzen) nur dann zulässig sei, wenn die am schlechtesten gestellte Bevölkerungsgruppe davon am meisten profitiert. ${ }^{17}$

Immer noch im utilitaristischen Rahmen bleibend, wurde von sozialwissenschaftlicher Seite mit der Theorie des rationalen Tauschs ${ }^{18}$ bzw. der strategischen Interaktion auf die Kritik an der analytischen Vernachlässigung der Verteilungsdimension reagiert. Beim rationalen Tausch können Individuen, die verschiedene Präferenzordnungen haben - z.B. A/B/C und $\mathrm{A} / \mathrm{C} / \mathrm{B}$ - und die zugleich über Ressourcen verfügen, die der $\mathrm{Be}-$ friedigung unterschiedlicher Bedürfnisse dienen können, über ihre Einsätze verhandeln und Ressourcen tauschen. ${ }^{19}$ Tausch kann so das Niveau der Bedürfnisbefriedigung aller Beteiligten erhöhen. Dieser Ansatz ist bei Fritz Scharpf zu einer Theorie strategischer Interaktion in Verhandlungssystemen erweitert worden; Verhandlungssysteme (negotiated agreement) gehören zu den (vier) grundlegenden Interaktionsmodi bzw. Formen sozialer Koordination. ${ }^{20}$

Auch im Modell strategischer Interaktion wird unterstellt, daß es um individuelle Nutzen geht, deren Summe zu maximieren ist. Das macht das hier in Anlehnung an Darstellungen bei Fritz Scharpf ${ }^{21}$ konstruierte Diagramm deutlich. Die beiden Koordinaten sind die individuellen Nutzen für $\mathrm{X}$ und für $\mathrm{Y}$; hier bringt Lösung $\mathrm{A}$ für $\mathrm{X}$ und Lösung B für Y den höchsten Nutzen. Der weiter nordöstlich liegende Punkt $C_{1}$ dagegen bringt eine höhere Nutzensumme als A oder B. Bei diesem Ansatz wird also zwischen individuellem Nutzen und Gesamtnutzen klar differenziert, wobei allerdings der Gesamtnutzen weiterhin eindeutig als Summe individueller Nutzen gemessen wird.

\footnotetext{
is Scharpf 1997, S. 90.

${ }^{16}$ Gaertner 1998.

17 Vgl. Rawls 1972, S. 302.

$18 \mathrm{Vgl}$. March/Olsen 1994.

19 Siehe dazu Coleman 1966.

${ }^{20} \mathrm{Vgl}$. Scharpf 1997.

21 Vgl. Scharpf 1993 u. 1997.
} 


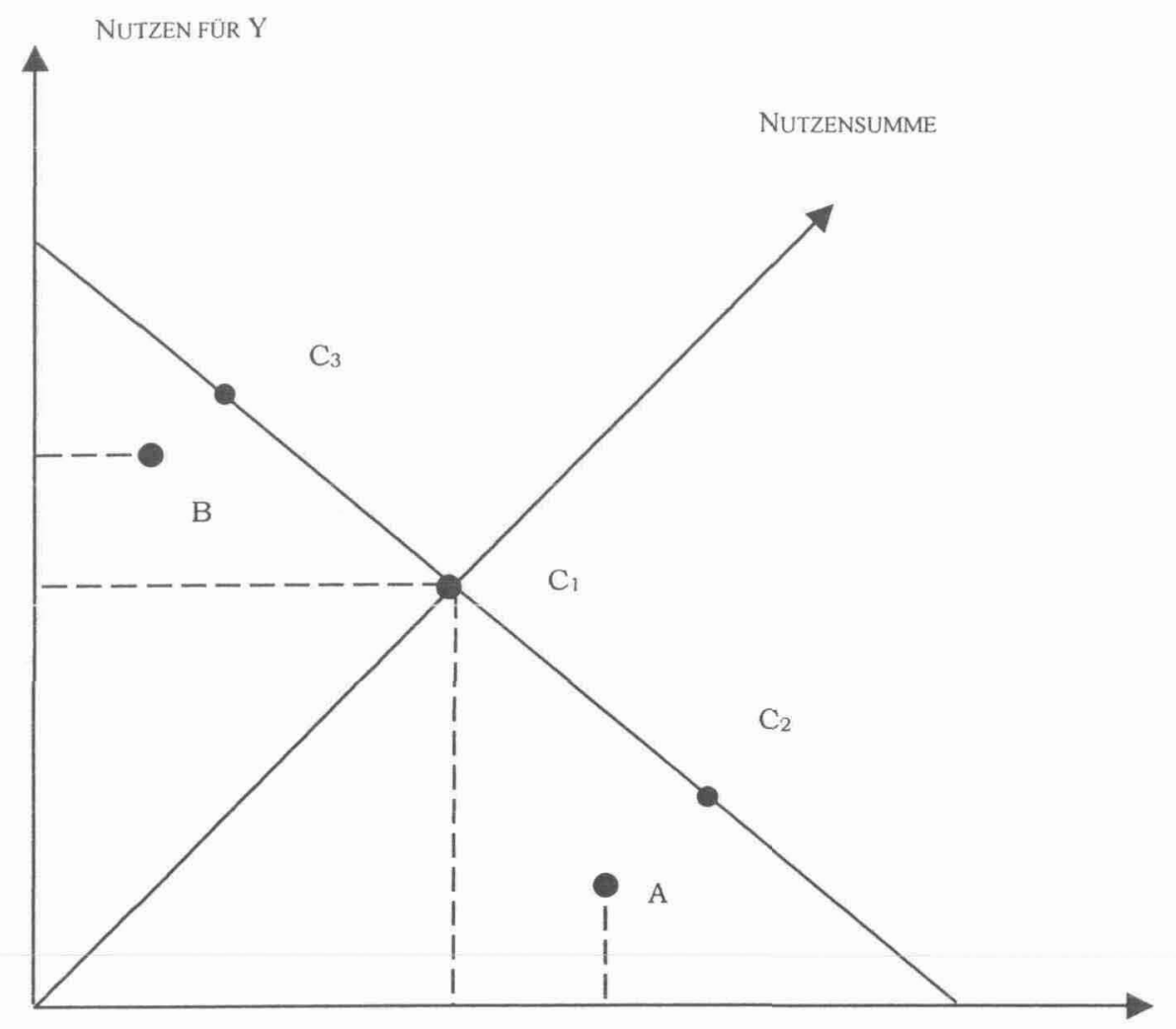

Abb. 1

NUTZEN FÜR X

Denken beide Spieler nur an die Maximierung ihres je individuellen Nutzens, würden sie - wie im berühmten Gefangenendilemma - die Punkte A bzw. B anstelle von $\mathrm{C}_{1}$ wählen; kooperieren sie, bekommen sie zusammen eine höhere Nutzensumme. Zieht man nun durch den Punkt des höchsten Gesamtnutzens, hier $\mathrm{C}_{1}$, eine von Nordwesten nach Südosten laufende Linie, dann hat man einen Nutzenisoquant (Coase) bestimmt; d.h. alle auf diesem Isoquanten liegenden Punkte haben den gleichen Gesamtnutzen. Es kommt also bei Verhandlungen zunächst darauf an, eine Lösung zu finden, die auf einem möglichst weit nach Nordosten hinaus geschobenen Isoquanten liegt, oder anders gesagt: Es geht um die Einigung auf den Punkt mit der höchsten erreichbaren Nutzensumme. Die verschiedenen Punkte auf einem Nutzenisoquanten unterscheiden sich jedoch hinsichtlich der Nutzenverteilung auf X und Y, und häufig ist keine Lösung mit gleicher Nutzenverteilung greifbar, sondern nur Lösungen mit ungleicher Nutzenverteilung. Wenn keine Lösung erreichbar ist, die beide Partner gleichermaßen befriedigt, kommt es zum Konflikt. 
Aber bei Verhandlungen gibt es eine Reihe von Möglichkeiten, entstandene Verteilungskonflikte zu lösen und damit die Einigung auf den Punkt mit dem höchsten erreichbaren Nettonutzen zu ermöglichen. Dazu gehören Ausgleichszahlungen, die Bündelung verschiedener Entscheidungen mit unterschiedlichen Nutzenprofilen und die Erweiterung des Zeithorizonts (zeitliche Entscheidungsbündelung). Alle diese Strategien setzen gegenseitiges Vertrauen voraus. Ist diese Voraussetzung gegeben, dann führt, wie Fritz Scharpf mit Hilfe einer Simulationsstudie demonstrieren konnte, ${ }^{22}$ die Kombination von Problemlösung (= Suche nach einem möglichst hohen Gesamtnutzen) und anschließender Lösung des Verteilungsproblems, d.h. eine Kombination von positiver und negativer Koordination, zu dem höchsten Nettonutzen von allen Konfigurationen, in denen die Spieler unterschiedliche Interessen bzw. Präferenzen in Bezug auf das Ergebnis haben. Ja, durch Verhandlung ist dann ein ebenso gutes Netto-Ergebnis zu erhalten wie im Idealfall hierarchischer Koordination, bei der ein weiser Hierarch die optimale Problemlösung oktroyiert.

\section{Gemeinwohl versus Nutzensumme}

Auch in der zuletzt beschriebenen, schon stark modifizierten Variante des utilitaristischen Ansatzes zur Bestimmung von Gemeinwohl wird von der Qualität der individuellen Bedürfnisse abstrahiert - und damit zugleich auch vom Inhalt des „Gesamtnutzens". Anders gesagt: Es gibt bei diesem Konzept nicht nur keinen Hinweis, daß der Gesamtnutzen an qualitativ anderen Maßstäben zu messen sein könnte als der Individualnutzen; die Summe der Individualnutzen bilden vielmehr unmittelbar die Komponenten oder Elemente, aus denen der Systemnutzen besteht: Je mehr aggregierte individuelle Bedürfnisbefriedigung, umso besser der Zustand des Systems. Dieses Postulat ist jedoch höchst fragwürdig. Private Güter können öffentliche Übel sein, wie Albert (1998) mit dem Hinweis auf - empirisch durchaus vorkommende - sadistische Bedürfnisse von Individuen bemerkt. Folgerichtig ist ein möglichst hoher Gesamtnutzen, eine möglichst hohe Summe individueller Bedürfnisbefriedigung nicht mit der Lösung eines Systemproblems gleichzusetzen. Um Systemprobleme bzw. deren Lösung zu bestimmen, braucht man einen eigenen Maßstab. In seiner kritischen Auseinandersetzung mit der von mir schon früher vertretenen Auffassung, ${ }^{23}$ daß zwischen der Notwendigkeit zur Erfüllung übergeordneter Systemimperative und der Befriedigung individueller Bedürfnisse unterschieden werden sollte, gibt Scharpf zwar zu, daß die Orientierung an Systemzielen auf der Ebene von Organisationen vorkommen kann, hält dies jedoch für selten und auf Grund sich immer stärker differenzierender Interessenpositionen und Situationsdeutungen zunehmend seltener werdend. Daß Individuen oder auch korporative Akteure sich am Systemnutzen der Gesellschaft orientieren könnten, bezweifelt er. Es ist also letztlich eine empirisch begründete Skepsis, wenn Scharpf auf einen eigenen Maßstab für Systembedürfnisse und Systemnutzen verzichtet und meint, die von den politischen Institutionen zu fördernde gemeinsame Wohlfahrt „,muss

22 Vgl. Scharpf/Mohr 1994.

$23 \mathrm{Vgl}$. Mayntz 1992. 
doch wohl aus den (wohlverstandenen) Interessen der (gegenwärtigen und künftigen) Mitglieder des Gemeinwesens abgeleitet werden - was, so denke ich, wohl oder übel auf eine utilitaristische Gemeinwohl-Definition hinausläuft".24

Betrachtet man allerdings diesen Satz, mit dem eine utilitaristische Definition von Systemnutzen als Nutzensumme individueller Bedürfnisbefriedigungen gerechtfertigt werden soll, genau, dann wird erkennbar, daß hier eben doch mit einem von dem aktuellen Nutzenstreben der Systemmitglieder verschiedenen Systemnutzen gearbeitet wird. Mit dem „wohlverstandenen“ und langfristigen, sogar künftige Generationen einbeziehenden Eigeninteresse wird Systemrationalität gewissermaßen über die Hintertür eingeführt. Allerdings, da Individualnutzen und Systemnutzen auf einer einheitlichen Skala gemessen werden (sonst wäre Addition nicht möglich), kann es dann bei der Bestimmung des individuellen Nutzens gar nicht um die Befriedigung aktuell tatsächlich empfundener Bedürfnisse der einzelnen Akteure gehen, sondern gewissermaßen um „vernünftige" Wünsche, um Wünsche, die bereits durch die Berücksichtigung der möglicherweise systemschädigenden Folgen ihrer Befriedigung diszipliniert sind.

In der Wirklichkeit entsprechen die je aktuellen und bei egoistischer Orientierung handlungsbestimmenden Präferenzen der Gesellschaftsmitglieder in vielen Fällen keineswegs ihren ,wohlverstandenen“ Interessen, die zwangsläufig die langfristige Sicherung eines zur individuellen Werteproduktion fähigen gesamtgesellschaftlichen Systems zum Inhalt haben müssen. Um die utilitaristische Qualität der Gemeinwohldefinition zu erhalten, bleibt also auch hier nur der Rückgriff auf eine ,unsichtbare Hand“, die aktuelle Präferenzen und, wohlverstandenes` Eigeninteresse zur Deckung bringt. Der mögliche Konflikt zwischen individuellen und systemischen Interessen wird auf diese Weise ausgeblendet. Sobald man dagegen den individuellen Nutzen nur hic et nunc und als real angestrebten misst, ist eine Diskrepanz zwischen individuellen und systemischen Bedürfnissen möglich und teilweise sogar wahrscheinlich. Ein positiver Systemzustand kann Werte maximieren müssen, die im Augenblick kein Systemmitglied für sich wünscht und die damit vom Wohlfahrtsmaximum aller Individuen auch bei einer längerfristigen Perspektive divergieren. Die Ultrastabilität eines Systems, also Systemrationalität, kann verlangen, daß individuelle Nutzen einer bestimmten Art weder heute noch morgen maximiert werden. Das aber heißt, daß individuelle Präferenzen problematisiert werden müssen, wenn man auf der Systemebene denkt. Auch in der Wohlfahrtsökonomie kommt man am Ende nicht darum herum, sich, wenn es konkret wird, zu fragen, was die gute Gesellschaft ist, und welche individuellen Präferenzen damit kompatibel sind.

Ein Verfahren, das aktuelle Präferenzen und langfristiges „wohlverstandenes“ Eigeninteresse, das auf systemische Qualitäten zielt, zur Deckung bringt, könnte der rationale Diskurs sein, wie ihn Rawls und Habermas konzipieren. Rawls gehört wie Habermas zu jenen, die annehmen, daß unter rationalen Menschen gesellschaftsweiter Konsens über die Gestaltung des Gemeinwesens erzielt werden kann, und zwar trotz divergierender Ausgangspunkte, wie z.B. Gerechtigkeitsvorstellungen. Solange allerdings ein solcher Diskurs wie bei Rawls nur dem rationalen Interessenausgleich dient, läuft er lediglich

${ }^{24}$ Scharpf 1994, S. 385. 
auf negative Koordination hinaus. Was das Rawls'sche Kriterium von Gerechtigkeit oder Fairness angeht, kann man zweifeln, ob es hier wirklich um Systemrationalität oder nicht doch nur um die Lösung von Verteilungskonflikten bei der individuellen Bedürfnisbefriedigung geht. Tatsächlich erkennt Rawls selber, daß es nicht ausreicht, wenn eine soziale Ordnung als gerecht akzeptiert wird. Ausdrücklich bemerkt er, daß es außer Gerechtigkeit noch andere Voraussetzungen für eine „viable human community“ gebe, und nennt dabei Koordination, Effizienz und Stabilität. ${ }^{25}$ Diese Aspekte von Systemrationalität behandelt Rawls jedoch nicht weiter.

Differenziert man, wie hier verlangt wird, zwischen Individualnutzen und Systemnutzen, dann stellt sich die Beziehung zwischen beiden als Kausalbeziehung dar, und es muß, ganz im Sinne theoretischer Ansätze, die sich mit der Mikro-MakroTransformation befassen, gefragt werden, wie sich ein bestimmtes Individualhandeln auf der Systemebene auswirkt. Dabei können sich bestimmte Formen egoistischen Handelns, wie von Adam Smith angenommen, auf der Systemebene positiv auswirken, etwa indem sie zu adaptiven Innovationen führen; Schumpeters Unternehmer wäre hier ein Beispiel. Andere Formen egoistischen Handelns, z.B. ein gesellschaftsweiter, ökonomisch oder mit Selbstverwirklichung begründeter Verzicht auf Reproduktion, kann für das System schädlich sein. Im Falle dysfunktionaler Präferenzen kann utilitaristische Wohlfahrtsmaximierung sogar den Systemtod bedeuten. Das gilt aber keineswegs nur für egoistisches, nur die aktuelle Bedürfnisbefriedigung suchendes Handeln. Auch bestimmte Formen „moralischen", sich an den jeweils sozial geltenden Normen orientierenden Handelns können systemschädigend sein; die Norm der Blutrache oder die exklusive Orientierung am Wohl der eigenen Familie wären offensichtliche Beispiele. Selbst normative Vorstellungen von Gerechtigkeit (etwa im Sinne erzwungener Gleichheit) und unmittelbar auf Gemeinwohl zielende Konzepte der Gesellschaftsgestaltung (etwa in Form von Planwirtschaft oder umgekehrt von ungezügelter Privatwirtschaft) können, wenn sie zur Leitlinie des Handelns werden, die Ultrastabilität eines gesellschaftlichen Systems und seine künftige Fähigkeit zur individuellen Werteproduktion beeinträchtigen. Genau diese Fähigkeit kann Institutionen und Leitlinien individuellen und kollektiven Handelns erfordern, die zu den aktuellen individuellen Präferenzen und Gemeinwohlkonzepten in Widerspruch stehen. Indem man dies als theoretische Möglichkeit einräumt, verabschiedet man sich nicht nur von utilitaristischen Ansätzen zur objektiven Gemeinwohlbestimmung, sondern auch von den eingangs erwähnten deliberativen Ansätzen der empirischen Sozialwissenschaft. Stattdessen braucht man dann aber einen unabhängigen Maßstab zur Beurteilung von Systemzuständen. Einen Versuch in dieser Richtung hat Talcott Parsons gemacht.

\section{Funktionalistische Systemtheorie}

Die Parsonssche strukturell-funktionale Systemtheorie scheint heute aus der wissenschaftlichen Diskussion verschwunden, und trotzdem liegt hier der vielleicht eindrucks-

${ }^{25}$ Vgl. Rawls 1972, S. 6. 
vollste und nachdrücklichste Versuch vor, so etwas wie Systemrationalität, einen Systemnutzen oder ein auf Gesellschaften als Ganze bezogenes Gemeinwohl zu bestimmen. Parsons interessierte sich zunächst, im Anschluss an die Entwicklung seiner Handlungstheorie ${ }^{26}$ für die Stabilität der Strukturen sozialer Systeme. ${ }^{27}$ Es ist oft darauf hingewiesen worden, daß dieses Interesse für soziale Ordnung als kritische Reaktion auf eine vom Utilitarismus geprägte Epoche zu verstehen sei. Parsons selbst hat sein Interesse für die von ihm immer als relativ betrachtete Stabilität sozialer Strukturen erkenntnistheoretisch begründet. Strukturen sind für Parsons relativ stabile Beziehungsmuster zwischen Akteuren, wobei die Stabilität der Beziehungen durch die normative Regulierung des Handelns erzeugt wird. Diese stabilisierende Regulierung beruht ihrerseits auf zwei Mechanismen, der Internalisierung von Normen im Prozess der Sozialisation, und der sozialen Kontrolle, die dem abweichenden Verhalten entgegenwirkt und es bändigt.

Parsons wollte jedoch niemals bei einer bloßen Struktur- oder Strukturierungsanalyse stehen bleiben. Sein Ziel blieb die dynamische Analyse, und hierzu eröffnete ihm der komplementäre Begriff der Funktion den Weg. Mit dem Begriffspaar Struktur und Funktion setzt Parsons eine analytische Tradition fort, die in der Soziologie schon von Comte begründet und von Spencer fortentwickelt wurde. Aber anders als bei Comte und Spencer wird der Funktionsbegriff bei Parsons zu einer Kategorie, die Struktur und Prozess übergeordnet ist. Mit Hilfe des Funktionsbegriffs will Parsons aus dem schier unübersehbaren Gewirr empirischer Phänomene das isolieren, was für das System wichtig, was systemrelevant ist. Um das aber sagen zu können, braucht man eine Vorstellung von einem bestimmten, als Ziel oder Maßstab dienenden Systemzustand. Dieser Zustand ist für Parsons zunächst die relative Stabilität, und die funktionale Analyse muß entsprechend fragen, wodurch Stabilität gewährleistet wird, d.h. was sozial-integrativ wirkt. Ganz ähnlich hatte schon Radcliffe-Brown Strukturerhaltung und Gewährleistung von Kontinuität als zentralen analytischen Bezugspunkt seines Funktionalismus gewählt. Parsons allerdings bezieht sich nicht auf den kulturanthropologischen Funktionalismus, sondern auf einen Aufsatz von Aberle et al., in dem vier Möglichkeiten des Zerfalls oder Untergangs von Systemen aufgezeigt werden: die biologische Auslöschung, die Apathie der Mitglieder, der Kampf aller gegen alle, oder das Aufgehen in einer anderen Gesellschaft. ${ }^{28}$ Die von Aberle u.a. dann entwickelten Funktionsvoraussetzungen sollen die Bedingungen darstellen, die es verhindern, daß ein soziales System auf eine dieser Weisen zugrunde geht. So werden Stabilität und Systemerhaltung bei Parsons gleichgesetzt.

Parsons erkennt jedoch, dass die Stabilitätsvoraussetzungen realer Gesellschaften nicht durch Prozesse auf der analytischen Ebene des von ihm ja recht eng definierten sozialen Systems allein erfüllt werden können; das ließe wohl nur soziale Kontrolle als Funktion übrig. Einen ersten Versuch, die Bedingungen systemischer Stabilität weiter zu fassen und gleichzeitig zu spezifizieren, machte Parsons mit dem Konzept des umfassenden Handlungssystems, in dem das soziale System nur noch eine von mehreren Ebenen bildet. Das Handlungssystem hat für Parsons zunächst drei, ab 1959 dann vier

26 Vgl. Parsons 1937.

27 Vgl. Parsons 1951.

28 Vgl. Parsons 1951, S. 26 ff. 
Ebenen, nämlich die Ebenen des Organismus, des Persönlichkeitssystems, des sozialen Systems und des kulturellen Systems. Die vier Systeme sind nicht aufeinander reduzierbar, sie sind aber interdependent, d.h. jede Systemebene hat für die anderen wichtige Funktionsvoraussetzungen zu erfüllen. Von zentraler Bedeutung ist dabei die Integration von Normen und Interessen, also vom kulturellen System und Persönlichkeitssystemen, im Prozess der Sozialisation.

Mit diesem Ansatz zur Bestimmung von Systemimperativen offenbar unzufrieden, entwickelt Parsons im letzten Schritt seiner Theorieelaboration ${ }^{29}$ ein Schema von vier Systemproblemen, die als Bezugspunkte einer funktionalistischen Analyse dienen können. Dies ist das berühmte AGIL-Schema (Anpassung, Zielerreichung, Integration und Aufrechterhaltung der Grundstrukturen). Dieses Schema gilt, wenn man z.B. die Fortentwicklungen bei Richard Münch betrachtet, wohl noch heute als Parsons' eigentlicher Beitrag zur funktionalistischen Systemtheorie. Dabei musste Parsons sein Augenmerk von der Differenzierung der Systemebenen und der Feststellung funktioneller Interdependenzen zwischen diesen aufs Ganze des Systems zurücklenken: die vier Systemfunktionen sind die wesentlichen funktionalen Imperative jedes umfassenden Handlungssystems, d.h. sie liegen als analytische Dimension quer zum Schema der Systemebenen. Außerdem wird jetzt die Bestandsproblematik nicht mehr auf den Aspekt der Integration und damit die Beherrschung systeminterner Spannungen reduziert. Das mehrere Ebenen umfassende Handlungssystem, das jede konkrete Gesellschaft darstellt, wird vielmehr als ein offenes System in einer Systemumwelt gesehen, an die es sich anpassen muß, wobei zu dieser Umwelt nicht nur andere Gesellschaften, sondern auch physische Gegebenheiten, die Natur usw. gehören. Parsons zentrale These ist, daß Anpassung, Zielerreichung, Integration und Aufrechterhaltung der Grundstrukturen die allgemeinsten Funktionsvoraussetzungen von Handlungssystemen sind, die deshalb auch permanent erfüllt werden müssen. Wieweit das tatsächlich geschieht, könnte als Maßstab des erzielten Systemnutzens, als Maßstab von Systemrationalität gelten.

Parsons hat sein Konzept der Systemfunktionen in einigen soziologischen Detailanalysen angewandt und dabei auf die systemische Funktionalität der Existenz beobachtbarer sozialer Strukturen oder auch Rollen hingewiesen. Im Zusammenhang mit seiner Theorie gesellschaftlicher Evolution ${ }^{30}$ legen diese Analysen die Interpretation nahe, daß sich Gesellschaften seiner Meinung nach gewissermaßen naturwüchsig in Subsysteme differenzieren, die sich auf die Erfüllung der vier Systemfunktionen spezialisieren. Die Anpassungsfunktion etwa wird vom Teilsystem Wirtschaft erfüllt, der Zielerreichung dient das Teilsystem Politik und der Aufrechterhaltung der Grundstrukturen u.a. das Bildungssystem. Parsons geht dabei auch nicht mehr vom Akteur, sondern vom System aus. Die Systemprobleme sind von ihm objektiviert worden, und die Handlungen der Akteure erscheinen als Produkte von Rollendefinitionen, die im Falle der Erfüllung der funktionellen Imperative auf systemische Problemlösung bezogen sind. Wenn Rollenerwartungen im Sozialisationsproze $\beta$ internalisiert sind, werden sie zur individuellen Bedürfnisdisposition, so daß am Ende die Verfolgung als privat wahrgenommener Ziele

\footnotetext{
29 Parsons/Smelser 1956.

30 Vgl. u.a. Parsons 1966.
} 
die kollektiven Werte realisiert. Die Individuen haben also im Idealfall eines positiven Systemzustands keine den Systeminteressen widersprechenden Bedürfnisse. Sie brauchen auch nicht zu wissen, daß ihr Handeln Systemfunktionen erfüllt, sondern sie tragen meist unabsichtlich zur Lösung von Systemproblemen bei (latente Funktionen).

Die Annahme einer in konkrete Gesellschaften eingebauten Tendenz zur Lösung von Systemproblemen ist unrealistisch und dient auch bei Parsons als abstrakter Grenzfall oder Idealfall. Aber genau diesen Idealfall und seine Voraussetzungen zu klären war sein Anliegen. Indem Parsons dabei auf der Integration von Persönlichkeitssystem und kulturellem System als Ordnungsvoraussetzung beharrt, erkennt er zwar implizit die Möglichkeit von Konflikten zwischen individuellem Nutzenstreben und Systembedürfnissen an. Er selber aber thematisiert diesen Konflikt nicht und operiert damit wie Adam Smith hypothetisch mit einer ,unsichtbaren Hand", die individuelle Bedürfnisse auf die Erfüllung verborgener Systemfunktionen hinlenkt. Auf diese Weise bleibt auch der systemtheoretische Ansatz faktisch eindimensional. Während es beim wohlfahrtsökonomischen Ansatz lediglich Konflikte um die Verteilung individuell appropriierbarer Nutzenquanten gibt, erkennt das Parsons'sche Modell nur z.B. aus organischen Dispositionen (Triebkräften) erwachsende Tendenzen zu abweichendem Verhalten an; für Auseinandersetzungen über die systemische Funktionalität bestimmter Rollenerwartungen bzw. Institutionen hat das Modell keinen Platz. Gleichzeitig bleibt der von Parsons angebotene Maßstab zur inhaltlichen Bestimmung von Gemeinwohl als Zustand der optimalen Erfüllung der vier Systemfunktionen viel zu abstrakt, um in Diskussionen über die Gemeinwohlimplikationen verschiedener Gesellschaftsverfassungen, Institutionen oder Handlungsorientierungen fruchtbar eingesetzt werden zu können.

\section{Bleibt Gemeinwohl unbestimmbar?}

Bleibt also, nachdem die beiden hier betrachteten Versuche einer inhaltlichen Gemeinwohlbestimmung sich als nicht tragfähig erwiesen haben, am Ende nichts anderes übrig, als sich auf einen prozeduralen Ansatz zurückzuziehen und für "Gemeinwohl" zu halten, was immer in einem kollektiven Meinungsbildungsprozeß oder von einer dazu legitimierten Instanz als Gemeinwohl definiert bzw. propagiert wird? Selbst der seinen eigenen analytischen Fähigkeiten gegenüber skeptische Sozialwissenschaftler braucht meines Erachtens eine derart radikale Konsequenz nicht zu ziehen.

Der zentrale Mangel der beiden hier betrachteten Ansätze zur Bestimmung von Gemeinwohl ist ihre Eindimensionalität. Mein Haupteinwand gegen den wohlfahrtsökonomischen Ansatz zur Gemeinwohlbestimmung ist, daß das Wohl des Ganzen als Ganzem - seine Ultrastabilität und seine Fähigkeit zur Werteproduktion für die Systemmitglieder - nicht mit der Erfüllung der je aktuellen Präferenzen der konkreten Systemmitglieder gleichgesetzt werden darf. So auch Kaufmann: „Kollektive Nutzen als Systemeffekte und individuelle Nutzen müssen heute als in zwei verschiedenen Dimensionen liegend behandelt werden; sie lassen sich nicht einfach verrechnen oder 
gar aufeinander reduzieren. ${ }^{\text {"31 }}$ Zwischen individuellem Handeln und den davon bestimmten Merkmalen des umfassenden gesellschaftlichen Systems besteht eine Kausalbeziehung, und dasselbe gilt für die Beziehung zwischen Systemmerkmalen und dem Ausmaß individueller Bedürfnisbefriedigung, die innerhalb eines solchen Systems möglich ist. Der individuelle Nutzen sollte als Folge, nicht als Komponente des Systemnutzens betrachtet werden. Das verlangt, systematisch zwischen Individualnutzen und Systemnutzen zu trennen und dabei zu berücksichtigen, daß beide nicht nur divergieren können, sondern oft sogar in unterschiedlichen Qualitäten bestehen. Systemstabilität, ökonomische Produktivität, Sicherheit der Systemgrenzen oder Erwartungssicherheit sind keine unmittelbar individuell appropriierbaren Güter, sondern emergente Systemmerkmale. Praktisch müßte sich das Interesse dann auf den Konflikt zwischen individueller und systemischer Rationalität und die Möglichkeiten konzentrieren, zwischen beiden zu vermitteln.

Aber wie soll der Systemnutzen als eine eigenständige Größe bestimmt werden? Hier kann Parsons' Versuch, ein abstraktes Konzept von Systemfunktionalität zu entwickeln, sicher nicht befriedigen. Jeder der Systemimperative - falls man einmal akzeptiert, daß es sich hier tatsächlich um die entscheidenden Funktionsvoraussetzungen handelt -, kann in einer konkreten Gesellschaft mehr oder weniger gut erfüllt sein, ohne daß man hier auch nur eine kritische Untergrenze der Funktionserfüllung genau bestimmen könnte. Und da es offensichtlich viele äquivalente institutionelle Lösungen für jedes grundsätzliche Systemproblem gibt, bietet Parsons' Konzept auch keine Grundlage für eine exakt vergleichende Beurteilung des jeweils in einer Gesellschaft verwirklichten Gemeinwohls. Das erreichbare Optimum auf Systemebene ist immer historisch kontingent: es besteht in einem Möglichkeitsraum, der durch Merkmale des Ist-Zustands eines Systems, sein Entwicklungs- und Selbststeuerungspotential begrenzt ist.

Dennoch sollte man nicht darauf verzichten, grundsätzlich von der eindimensionalen Betrachtungsweise wohlfahrtsökonomischer Ansätze auf eine mehrdimensionale Betrachtungsweise überzugehen. Der empirisch-analytisch orientierte Sozialwissenschaftler wird dabei nicht auf die utopische Vorstellung zurückgreifen, daß ein rationaler Diskurs unter allen Gesellschaftsmitgliedern zur Einsicht in die Eigenschaften und Voraussetzungen eines positiven Systemzustands führt. Als Ausgangspunkt bietet sich die Tatsache an, daß Individuen über eine abgestufte Reihe sozialer Identitäten verfügen. Sie können sich mit der Familie, einer Kleingruppe, der Siedlungsgemeinschaft oder einer sozialen Klasse und unter bestimmten Voraussetzungen sogar mit ihrer - national verfaßten - Gesellschaft identifizieren. Auf der Grundlage der zuletzt genannten Identität können Individuen als Bürger und mehr noch als eigens hierfür bestellte Politiker darüber nachdenken, was im Interesse des Ganzen zu tun wäre - wobei die Akteure durchaus wahrnehmen können, daß das, was dem Gemeinwesen dient, nicht zugleich ihren eigenen Interessen dienen muß. Empirisch wären dann die Inzidenz und die Voraussetzungen zu untersuchen, unter denen die verschiedenen Orientierungen handlungsbestimmend werden, und wie dabei auftretende Konflikte gelöst oder auch nicht gelöst werden. Hierzu gibt es in der Forschung durchaus Ansätze, z.B. wenn man fragt, unter welchen Umständen in einem Verhandlungssystem eine Problemlösungsorientie-

${ }^{31}$ Kaufmann 1994, S. 365. 
rung dominiert und Verteilungsprobleme (Aspekte partikularer Interessenvertretung) in den Hintergrund rücken. Am Beispiel der Transformation der Akademie der Wissenschaften der DDR habe ich einige dieser Voraussetzungen analysiert. ${ }^{32}$

Aber mit der Dominanz einer Problemlösungsperspektive allein ist es nicht getan. Nicht alle auf dem Wege der Auseinandersetzung und Kompromißbildung gefundenen Gemeinwohlziele lassen sich als gleichwertig im Sinne von Systemrationalität betrachten. Zwar machen ein historischer Rück- und ein Seitenblick auf gegenwärtig existierende Gesellschaften klar, daß es keine einzige konkrete Gesellschaftsform gibt, die sich unter der Perspektive von Systemrationalität als ideal bezeichnen ließe. Aber wir können doch etwas mehr, als absolute Minimalbedingungen für die Überlebensfähigkeit konkreter Gesellschaften und ihre Fähigkeit zur individuellen Wohlfahrtsproduktion formulieren. Es gibt keinen guten Grund dafür, daß sich die Sozialwissenschaft in dieser Hinsicht abstinent verhält und selbst die Theorie politischer Steuerung weitgehend darauf verzichtet, die Problemdiagnosen, die sie als Bezugspunkt ihrer Analyse politischer Entscheidungsprozesse nimmt, kritisch zu beleuchten. Warum sollte die Sozialwissenschaft unfähig sein, Ähnliches zu leisten wie die Naturwissenschaften bei der Erforschung von Organismen oder von Ökosystemen?

Tatsächlich gibt es auch in den Sozialwissenschaften (und nicht nur in der Ökonomie!) Ansätze, die sich nicht damit bescheiden, als Systemproblem zu akzeptieren, was in irgendwelchen kollektiven Meinungsbildungsprozessen oder von einer politischen Instanz als solches definiert wird. Sicher wird man dabei zwischen den Gefahren des Identitätsverlusts (Zerfallen einer bestimmten Gesellschaftsordnung), der Regression auf frühere Entwicklungsstufen und der physischen Auslöschung unterscheiden wollen, wobei sich Versuche physischer Auslöschung, etwa durch massenhaften wechselseitigen Totschlag im Gefolge ethnischer oder religiöser Konflikte oder durch Eroberung und Absorbierung in eine andere Gesellschaft (wie es z.B. den der physischen Vernichtung entgangenen amerikanischen Indianern geschah), auch als Identitätsverluste manifestieren. Hier hat die Konflikt- und Friedensforschung einige Einsichten zu bieten. Weiter wird man vielleicht mit David Lockwood (1979) zwischen dem erreichten Grad an Sozialintegration und Systemintegration, d.h. der Abstimmung verschiedener funktioneller Teilsysteme aufeinander, unterscheiden wollen. Ohne Zweifel macht dabei die Tendenz zur funktionellen Differenzierung die Definition von Problemen auf der Ebene des gesamten Systems schwierig. Trotzdem sind tragfähige Problemdiagnosen zumindest auf Teilsystemebene möglich, wie nicht zuletzt die Untersuchungen zur Frage der Zukunft des „Sozialstaats“, des etablierten Systems sozialer Sicherung, oder über Ursachen und Lösungsmöglichkeiten der „Beschäftigungskrise“ zeigen. Auch ist es, wie z.B. bei der gegenwärtigen Beschäftigungskrise, durchaus möglich, Wege zur Problemlösung aufzuweisen - wobei sich interessante Unterschiede in der Problemlösungsfähigkeit verschiedener nationaler Gesellschaften zeigen.

Es kann nicht der Zweck dieser wenigen Zeilen sein, über die beiden diskutierten Ansätze hinausgehend ein eigenes Konzept für den sozialwissenschaftlichen Umgang mit dem theoretischen Gemeinwohlproblem zu entwickeln. Die sich heute akzentuierende Gemeinwohldebatte sollte aber die Sozialwissenschaften stimulieren, sich stärker

\footnotetext{
32 Vgl. Mayntz 1994, S. 272-281.
} 
mit der Diagnose von Systemproblemen zu beschäftigen, um so bei der Analyse von sozio-politischen Willensbildungsprozessen, Selbststeuerungseffekten und Aggregateffekten einen Maßstab zu haben, um den Erfolg oder Mißerfolg deliberativer Formen der Gemeinwohlbestimmung beurteilen zu können.

\section{Literaturverzeichnis}

Albert, H. (1998), Bemerkungen zur Wertproblematik. Von der Bewertung des Sozialprodukts zur Analyse der sozialen Ordnung, in: Lectiones Jenenses, H. 15.

Alemann, U. v./Heinze, R. G./Wehrhöfer, U. (Hg, 1999), Bürgergesellschaft und Gemeinwohl. Analyse. Diskussion. Praxis, Opladen.

Blickle, P. (2001), Der Gemeine Nutzen. Ein kommunaler Wert und seine politische Karriere, in: Gemeinwohl und Gemeinsinn. Historische Semantiken politischer Leitbegriffe, hg. v. H. Münkler/H. Bluhm, Berlin, S. 85-107.

Coleman, J. S. (1966), Foundations for a Theory of Collective Decisions, in: AJS 71, S. 615-627.

Derlin, H.-U./Gerhardt U./Scharpf, F. W. (Hg., 1994), Systemrationalität und Partialinteresse, BadenBaden.

Gaertner, W. (1998), Armut und Verteilungsgerechtigkeit, in: Spektrum der Wissenschaft, Dez., S. 30-34.

Kaufmann, F.-X. (1994), Staat und Wohlfahrtsproduktion, in: Systemrationalität und Partialinteresse, hg. v. H.-U. Derlin/U. Gerhardt/F. W. Scharpf, Baden-Baden, S. 357-380.

Lockwood, D. (1979), Soziale Integration und Systemintegration, in: Theorien des sozialen Wandels, hg. v. W. Zapf, Königstein, S. 124-140.

March, J./Olsen J. (1994), Institutional Perspectives on Governance, in: Systemrationalität und Partialinteresse, hg. v. H.-U. Derlin/U. Gerhardt/F. W. Scharpf, Baden-Baden, S. 249-270.

Mayntz, R. (1992), Interessenverbände und Gemeinwohl - Die Verbändestudie der Bertelsmann Stiftung, in: Verbände zwischen Mitgliederinteressen und Gemeinwohl, hg. v. dies., Gütersloh, S. 11-35.

Mayntz, R. (1994), Deutsche Forschung im Einigungsprozeß. Die Transformation der Akademie der Wissenschaften der DDR 1989 bis 1992, Frankfurt/M./New York.

Mayntz, R. (1997), Policy-Netzwerke und die Logik von Verhandlungssystemen, in: Soziale Dynamik und politische Steuerung. Theoretische und methodologische Überlegungen, hg. v. R. Mayntz, Frankfurt/M./New York, S. 239-262.

Münkler, H./Fischer K. (1999), Gemeinwohl und Gemeinsinn. Thematisierung und Verbrauch soziomoralischer Ressourcen in der modernen Gesellschaft, in: Berlin-Brandenburgische Akademie der Wissenschaften: Berichte und Abhandlungen, Bd. 7, Berlin, S. 237-265.

Nunner-Winkler, G. (1994), Zur Frage der Moral in der Politik - eine Frage des Systems oder der Persönlichkeit?, in: Systemrationalität und Partialinteresse, hg. v. H.-U. Derlin/U. Gerhardt/F. W. Scharpf, Baden-Baden, S. 123-149.

Offe, C. (2001), Wessen Wohl ist das Gemeinwohl?, in diesem Band.

Parsons, T. (1937), The Structure of Social Action, New York.

Parsons, T. (1951), The Social System, Glencoe III.

Parsons, T. (1966), Societies: Evolutionary and Comparative Perspectives, Englewood Cliffs.

Parsons, T./Smelser N. J. (1956), Economy and Society, New York.

Rawls, J. (1972), A Theory of Justice, Oxford.

Scharpf, F. W. (1993), Coordination in Hierarchies and Networks, in: Games in Hierarchies and Networks. Analytical and Empirical Approaches to the Study of Governance Institutions, hg. v. ders., Frankfurt/M./New York, S. 125-165. 
Scharpf, F. W. (1994), Politiknetzwerke als Steuerungssubjekte, in: Systemrationalität und Partialinteresse, hg. v. H.-U. Derlin/U. Gerhardt/F. W. Scharpf, Baden-Baden, S. 381-402.

Scharpf, F. W. (1997), Games Real Actors Play. Actor-Centered Institutionalism in Policy Research, Boulder, Col.

Scharpf, F. W./Mohr M. (1994), Efficient Self-Coordination in Policy Networks, MPIfG Discussion Paper 1.

Schuppert, G. F. (2002), Gemeinwohldefinition im kooperativen Staat, in: Gemeinwohlkonkretisierungen und Gemeinsinnerwartungen im Recht, hg. v. H. Münkler/K. Fischer, Berlin (i.E.). 